of Scleroderma and its complications: Kymera Therapeutics, Mitsubishi Tanabe, Thomas Frauenfelder: None declared, Stephanie Tanadini-Lang: None declared, Britta Maurer Speakers bureau: Speaker fees from Boehringer-Ingelheim, Grant/ research support from: Grant/research support from AbbVie, Protagen, Novartis Biomedical Research, congress support from Pfizer, Roche, Actelion, mepha, and MSD

DOI: 10.1136/annrheumdis-2021-eular.2884

\section{POS0867 ALTERATION OF DUODENAL HISTOLOGY IN SYSTEMIC SCLEROSIS PATIENTS AFTER FECAL MICROBIOTA TRANSPLANTATION}

H. Didriksen ${ }^{1}$, N. Strahm ${ }^{2}$, Ø. Molberg ${ }^{1}$, H. Fretheim ${ }^{1}$, T. Garen ${ }^{1}$, Ø. Midtvedt ${ }^{1}$, O. Distler ${ }^{2}$, G. Kania ${ }^{2}$, A. M. Hoffmann-Vold ${ }^{1} .{ }^{1}$ Oslo universitetssykehus Rikshospitalet, Rheumatology, Oslo, Norway; ${ }^{2}$ University Hospital of Zürich, Rheumatology, Zürich, Switzerland

Background: Systemic sclerosis (SSc) is a complex autoimmune, multi-organ disease with gastro-intestinal tract (GIT) involvement significantly contributing to comorbidity. While understanding of mechanisms behind SSc-related GIT disease is incomplete, recent work shows that altered gut microbiota (dysbiosis) is present in many patients and associates with specific GIT symptoms. Intending to improve dysbiosis, we set up the controlled ReSScue pilot trial, where fecal microbiota transplantation (FMT) was conducted by duodenal infusions of Anaerobic Cultivated Human Intestinal Microbiome (ACHIM) or placebo. The results indicated that FMT by ACHIM induced short-term improvement on patient reported GIT symptoms, as evaluated by the validated UCLA GIT score (1). Duodenal biopsies were taken before and after FMT to assess potential effects of ACHIM on small intestinal mucosa.

Objectives: Assessment of duodenal histology and GIT symptoms before and after FMT by ACHIM compared to placebo.

Methods: In this explorative study, we assessed duodenal biopsies collected prior to first FMT (week 0), prior to second FMT (week 2) and at study end (week 16) from ReSScue trial patients who either received ACHIM $(n=5)$ or placebo $(n=4)$. To examine potential effects of FMT on the duodenal mucosa, we performed immunohistochemistry (IHC) staining on paraffin-embedded tissue samples using the following markers: Sirius red (SIR) for collagen fibers and monoclonal antibodies against gp38 (podoplanin, as marker for lymphatic vessels and fibroblasts), CD38 (as preliminary marker for adaptive immune cells) and CD64 (as preliminary marker for innate immune cells). We determined staining per area in the individual tissue slides for each marker using ImageJ Fiji. The mean total UCLA GIT score for the patients were calculated at week 0,2 and 16, as well as the mean staining per area of the duodenum biopsies at week 0,2 and 6 for all the markers. Finally, correlations between mean staining per area and mean total UCLA GIT score were assessed for ACHIM and placebo patient groups by Pearson correlation ( $r$ ).

Results: All nine patients included in the pilot trial were female and had limited cutaneous SSc. Groups receiving ACHIM and placebo had comparable disease duration (1). We found that relative change in staining per area for SIR from week 0 to week 2 differed between the ACHIM group and the placebo group (Figure $1 \mathrm{~A}$, left panel). Similar changes were observed for anti-gp38, anti-CD38 and anti-CD64 (left panel in Figures 1B-D). Strong correlations were found between mean total UCLA GIT score and the mean staining per area of the markers SIR ( $r=0.98)$, anti-gp38 ( $r=0.94)$, anti-CD34 ( $r=0.85)$ and CD64 ( $r=0.93)$ in the ACHIM group (Figure 1, A-D). In the placebo group, there was no correlation between the UCLA GIT score and anti-gp38 $(r=0.22)$ and anti-CD64 ( $r=0.21)$, however, a strong correlation were observed to $\mathrm{SIR}(\mathrm{r}=0.86)$ and anti-CD38 ( $r=0.92)$ staining (Figure 1A-D, right panel).

Conclusion: This explorative data set indicates different effects of FMT by ACHIM and placebo on the duodenal mucosa of SSc patients with GIT affection. Interestingly, we observed correlations between mucosal markers and improved patient reported GIT symptoms in the ACHIM group.

REFERENCES

[1] Fretheim H, Chung BK, Didriksen H, Bækkevold ES, Midtvedt Ø, Brunborg $\mathrm{C}$, et al. Fecal microbiota transplantation in systemic sclerosis: A double-blind, placebo-controlled randomized pilot trial. PLoS One. 2020;15(5):e0232739.
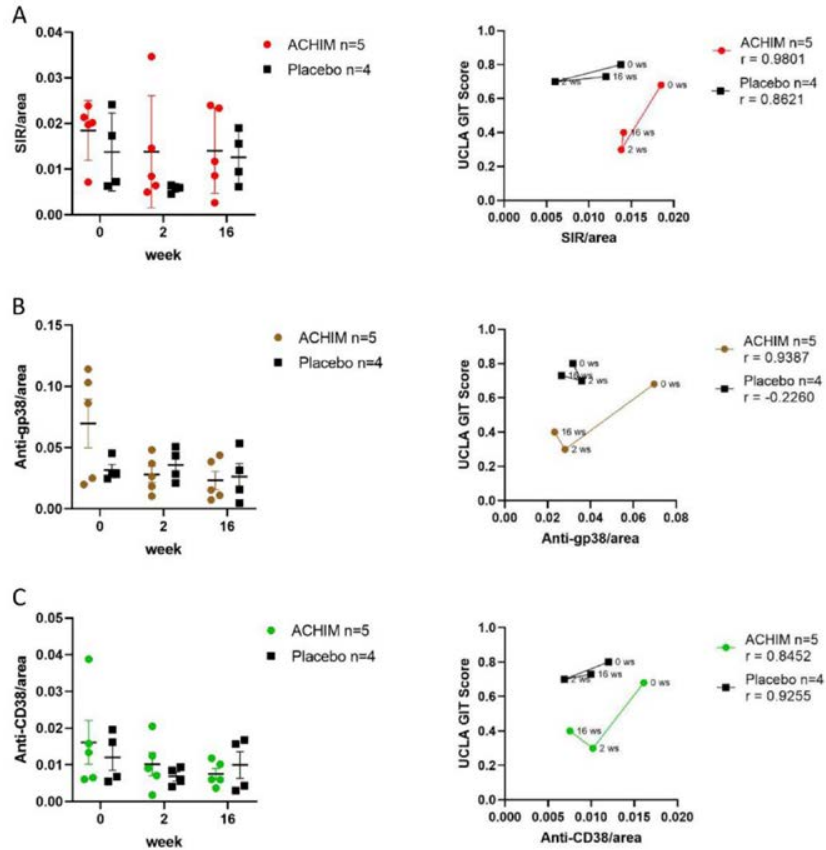

D
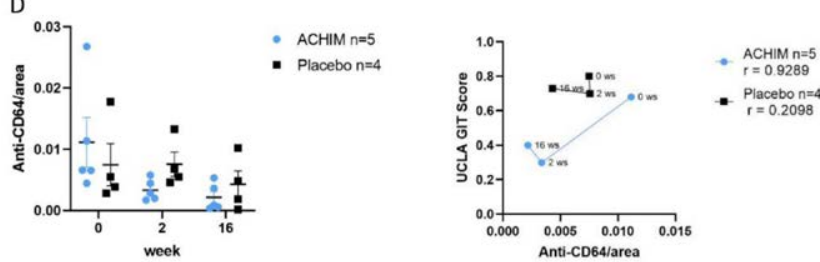

Figure 1. Relative staining per area in ACHIM and placebo group (left panel) and correlation between total UCLA GIT score and staining ratios at time point 0,2 and 16 weeks in patients receiving $\mathrm{ACHIM}$ and placebo (right panel).

Acknowledgements: We will like to thank Maria Comazzi for all the work she have done with the IHC stainings

Disclosure of Interests: Henriette Didriksen Speakers bureau: Travel bursary - GSK

Travel bursary and speaker - Actelion, Noemi Strahm: None declared, Øyvind Molberg: None declared, Håvard Fretheim Speakers bureau: Received travel bursaries from Actelion, and remuneration from Bayer., Torhild Garen: None declared, Øyvind Midtvedt: None declared, Oliver Distler Speakers bureau: Actelion, Kymera Therapeutics, Mitsubishi Tanabe Pharma, Abbvie, Acceleron, Alexion, Amgen, AnaMar Arxx Therapeutics, Baecon Discovery, Blade Therapeutics, Corbus Pharmaceuticals, Drug Development International Ltd, CSL Behring, Galapagos NV, Glenmark Pharmaceuticals, GSK, Horizon (Curzion) Pharmaceuticals, Inventiva, iQvia, Kymera Therapeutics, Lilly, Novartis, Pfizer, Topadur and UCB, Grant/research support from: Boehringer Ingelheim, Beyer, Gabriela Kania: None declared, Anna-Maria Hoffmann-Vold Speakers bureau: Actelion, Roche, Bayer, Merck Sharp \& Dohme, Lilly, ARXX and Medscape, Grant/research support from: Boehringer Ingelheim DOI: 10.1136/annrheumdis-2021-eular.2936

\section{\begin{tabular}{|l|l}
\hline POS0868 THE FORMULA TO PREDICT TACROLIMUS \\
\hline
\end{tabular} CONCENTRATION ACCORDING TO GENOTYPING OF CYP3A5 IS USEFUL FOR EFFECTIVE TREATMENT IN INTERSTITIAL LUNG DISEASE WITH DERMATOMYOSITIS}

M. Katayama ${ }^{1}$, S. Horibata ${ }^{2,3}$, S. Takahashi ${ }^{1,3}$, M. Takahashi ${ }^{3}$, T. Saito ${ }^{3}$, N. Amano ${ }^{1}$, K. Yoshida ${ }^{1}$, S. Hatachi ${ }^{1}$, K. Yorifuji ${ }^{2,3}$, S. Kumagai ${ }^{1,3}{ }^{1}$ Shinko Hospital, Center for Rheumatic Disease, Kobe, Japan; ${ }^{2}$ Shinko Hospital, Department of Pharmacy, Kobe, Japan; ${ }^{3}$ Shinko Institute for Medical Research, Kobe, Japan 